\title{
Announcement: Social Media Editor
}

JPRM is looking to recruit a social media editor! We are hoping to create social media profiles on Twitter and Facebook in order to promote the journal, our content, and the authors of manuscripts we publish. This is an excellent opportunity to develop science communication and networking skills.

This is a volunteer position and may be invaluable to early-career investigators. If you are interested, please email jprmbss@gmail.com and epico@mail.cho.org with a one-page description of your qualifications and interest in the position.

Social media editor description:

- Promote the journal through regular social media posts

- Editing and creation of photo, video, and audio content for social media promotion

- Interview authors to share research experiences, techniques, and results

- Network with other organizations relevant to pediatrics, rehabilitation, patient and family support groups

- Collaborate with members of the editorial staff and board

- Brainstorm and plan themed issue topics for future publications

- (optional) Possibility to edit manuscripts and contribute to the overall manuscript publishing process

Necessary qualifications:

- Familiarity with Facebook, Twitter, Youtube, and other social media platforms

- Basic editing techniques with photo, video, and audio

Bonus qualifications:

- Knowledge of research publishing process

- Experience producing photo, video, and audio content

- Expertise with pediatric rehabilitation topics 